\title{
A Data-Driven Approach to Simulate Pedestrian Competitiveness Using The Social Force Model
}

\author{
Geng Cui ${ }^{1}$. Daichi Yanagisawa ${ }^{2,3,4}$. Nishinari Katsuhiro ${ }^{2,3,4}$ \\ ${ }^{1}$ Department of Advanced Interdisciplinary Studies, Graduate School of Engineering, \\ The University of Tokyo, Tokyo, Japan \\ E-mail: cuigeng@g.ecc.u-tokyo.ac.jp \\ 2 Research Center for Advanced Science and Technology, The University of Tokyo, Tokyo, Japan \\ ${ }^{3}$ Department of Aeronautics and Astronautics, School of Engineering, \\ The University of Tokyo, Tokyo, Japan \\ ${ }^{4}$ Mobility Innovation Collaborative Research Organization, The University of Tokyo, Chiba, Japan \\ E-mail: tDaichi@mail.ecc.u-tokyo.ac.jp, tknishi@mail.ecc.u-tokyo.ac.jp
}

Received: 12 August 2021 / Last revision received: 4 November 2021 / Accepted: 4 November 2021 DOI: $10.17815 / \mathrm{CD} .2021 .118$

\begin{abstract}
The research of pedestrian evacuation dynamics is of significance for understanding and preventing human stampedes. Since the experimental approach of reproducing true emergency evacuations is impossible due to safety issues. The theoretical approach based on numerical simulation has called the attention of researchers. In the simulation of pedestrian evacuation, a critical problem is how to simulate pedestrian competitiveness to reproduce emergency evacuation. Based on the social force model, researchers have tried to simulate pedestrian competitiveness by adjusting some model parameters. However, in most cases handcrafted values are adopted without calibration, thus unrealistic results might be produced.

In this study, we applied a differential evolutionary algorithm to determine the optimal parameter specifications of the social force model by adjustment to experimental data. We conducted pedestrian experiments where five participants including patient and impatient individuals proceeded through a narrow corridor. Taking the distance between simulation results and experimental data as the objective function, a minimization problem was generated. A differential evolutionary algorithm was adopted to search for the optimal combination of parameters. We found that though at initialization all the parameter values were randomly determined, the difference between patient and impatient pedestrians could be captured by adjustment to experimental data. This highlights the need to better understand and research pedestrian heterogeneity in terms of competitiveness.
\end{abstract}


Keywords Evolutionary optimisation - social force model · pedestrian simulation . crowd evacuation $\cdot$ pedestrian competitiveness

\section{Introduction}

Pedestrian evacuation has attracted considerable research interest in the last years. A large body of experimental studies was devoted to investigating collective dynamics during the evacuation process [1]. However, due to safety concerns, conducting evacuation experiments in a real emergency is impossible, thus it is hard to study the system with a high degree of realism by the experimental approach.

Numerical simulation is another approach that does not have to deal with the difficulties of the experimental approach. The social force model is one of the most well-applied pedestrian models [2], based on which collective phenomena including lane formation, oscillations at bottlenecks, and clogging at exit doors were reproduced [3][4].

In the application of the social force model, a well-used method to simulate pedestrian competitiveness is adjusting some model parameters. The existing approaches are limited by at least one of the following shortcomings: (i) Handcrafted parameter values are adopted and therefore unrealistic results might be produced [5][6]. (ii) Only a part of the parameters are calibrated [7][8], it is worth mentioning that a strong correlation exists in the parameter space. (iii) The model is calibrated in a situation with a specific competitiveness level [9]. Although the results are promising, these studies are not adaptive to other situations with different competitiveness levels.

In this study, we overcome the aforementioned limitations with a heuristic data-driven approach to determine the optimal parameters combination of the social force model. This study aims to investigate how to represent pedestrian heterogeneity in terms of competitiveness based on the social force model. We conducted a small-scale pedestrian experiment, where a group of heterogeneous pedestrians proceeded through a narrow bottleneck. Taking experimental data including egress time and positions of pedestrians extracted from the experiment video as reference data, we generate a minimization problem of which the objective function is the distance between simulation results and the reference data. A differential evolutionary algorithm (DE) is adopted to search for a set of parameters that enables the best match between the simulation results and the reference data.

The rest of the paper is organized as follows. In Sec. 2 we give a review of related works. Sec. 3 describes the pedestrian experiment from which the reference data was extracted and our data-driven framework for parameter optimisation. In Sec. 4 the results and discussion are presented. Finally, Sec. 5 is devoted to the conclusion and future study. 


\section{Related Work}

\subsection{Experimental studies of competitive and non-competitive evacuation}

In the study of pedestrian evacuation dynamics, the compact of pedestrian competitiveness is one of the most studied topics. A large body of experimental studies is contributed to examine the 'faster-is-slower' effect, which refers to the phenomenon of a statistical decrease in evacuation efficiency as pedestrians push harder through a bottleneck. However, there is a mixture of evidence in the literature [1]. On one hand, the 'faster-is-slower' effect is observed in pedestrian experiments. A. Garcimartín et al. conducted pedestrian experiments in two situations: non-competitive evacuation and competitive evacuation, it is found that in the competitive situation, the evacuation time is longer [10]. J. M. Pastor et al. investigated bottleneck flow for three different kinds of discrete particles: human, sheep, and grains. In the experiments using human and sheep objects, it is observed that high competitiveness results in longer time lapses between consecutive egress [11]. On the other hand, there are studies contradicting the 'faster-is-slower' effect. According to experiments conducted with a mixture of population under three different stress levels, the authors suggested that 'a higher stress level leads to a higher speed and a higher capacity' [12]. In experiments conducted by another research group, three different levels of competitiveness along with varying doorway widths are considered, the results indicate that faster was invariably faster [1]. The reason of such mixed results might be that pedestrian competitiveness is hard to quantitatively control, the level of competitiveness differs according to different research groups.

\subsection{Pedestrian competitiveness simulation}

Based on the social force model, researchers have tried to represent pedestrian competitiveness using parameters representing pedestrian properties such as desired velocity, the strength of interaction, and individual relaxation time. The 'faster-is-slower' effect was initially proposed by assigning different desired velocities to pedestrians [4]. The same approach was adopted by another group to simulate competitive and cooperative egress behaviour [13]. Furthermore, the spatial game theory was incorporated with the social force model to represent strategic interactions [14][15]. However, these studies are all based on handcrafted values, e.g. it is assumed that the desired velocity of patient pedestrians is lower than impatient pedestrians, and the strength of interaction of patient pedestrians is stronger, to what extent is such assumption correct is still not verified.

\subsection{Parameter calibration}

Up to now, several parameter calibration approaches have been proposed. e.g. maximum likelihood estimation, simulated annealing, genetic algorithm, and differential evolutionary algorithm [2]. Generally, the parameters are calibrated toward reducing the distance 


\begin{tabular}{|c|c|c|c|c|c|}
\hline Year & Researcher & Data type & Reference data & $\begin{array}{l}\text { Updating algo- } \\
\text { rithm }\end{array}$ & Calibrated parameter \\
\hline 2007 & $\begin{array}{l}\text { A. Johansson et } \\
\text { al. [7] }\end{array}$ & $\begin{array}{l}\text { Empirical data of a real life } \\
\text { uncompetitive situation }\end{array}$ & $\begin{array}{l}\text { Pedestrian trajec- } \\
\text { tory }\end{array}$ & $\begin{array}{l}\text { Simple evolution- } \\
\text { ary algorithm }\end{array}$ & $\begin{array}{l}\text { Interaction strength, } \\
\text { Interaction distance, } \\
\text { Anisotropy parameter }\end{array}$ \\
\hline 2015 & M. Li et al. [9] & $\begin{array}{l}\text { Empirical data of the } 2013 \\
\text { Ya'an earthquake }\end{array}$ & $\begin{array}{l}\text { Number of evac- } \\
\text { uated people } \\
\text { against time }\end{array}$ & $\mathrm{DE}$ & $\begin{array}{l}\text { Desired velocity, Interac- } \\
\text { tion strength, Interaction } \\
\text { distance, Friction coeffi- } \\
\text { cient, Compression coeffi- } \\
\text { cient }\end{array}$ \\
\hline 2015 & $\begin{array}{l}\text { J. Zhong et al. } \\
{[17]}\end{array}$ & $\begin{array}{l}\text { Empirical data of a pedes- } \\
\text { trian crossing, Simulation } \\
\text { results based on prede- } \\
\text { fined parameter values }\end{array}$ & $\begin{array}{l}\text { Pedestrian den- } \\
\text { sity distribution }\end{array}$ & $\begin{array}{l}\text { DE, Genetic al- } \\
\text { gorithm, DE with } \\
\text { global and local } \\
\text { search modules }\end{array}$ & $\begin{array}{l}\text { Four parameters of the so- } \\
\text { cial force model and some } \\
\text { scenario specific parame- } \\
\text { ters }\end{array}$ \\
\hline 2015 & $\begin{array}{l}\text { J. Zhong and W. } \\
\text { Cai [18] }\end{array}$ & $\begin{array}{l}\text { Empirical data of a pedes- } \\
\text { trian crossing, Simulation } \\
\text { results based on prede- } \\
\text { fined parameter values }\end{array}$ & $\begin{array}{l}\text { Pedestrian den- } \\
\text { sity distribution }\end{array}$ & $\begin{array}{l}\text { DE with sensi- } \\
\text { tivity analysis } \\
\text { and the Powell's } \\
\text { method }\end{array}$ & $\begin{array}{l}\text { Seven parameters of the } \\
\text { social force model and one } \\
\text { scenario specific parame- } \\
\text { ter }\end{array}$ \\
\hline 2021 & $\begin{array}{l}\text { I. M. Sticco et al. } \\
\text { [8] }\end{array}$ & $\begin{array}{l}\text { Empirical data of a high } \\
\text { stress event }\end{array}$ & $\begin{array}{l}\text { Pedestrian egress } \\
\text { time }\end{array}$ & $\mathrm{DE}$ & $\begin{array}{l}\text { Friction coefficient, Com- } \\
\text { pression coefficient }\end{array}$ \\
\hline
\end{tabular}

Table 1 Review on the parameter calibration studies

between simulation results and reference data. Well-used reference data include pedestrian trajectory and density distribution, from a microscopic and macroscopic perspective of view, respectively [16]. Since the relationship between parameter values and simulations results is hard to explicitly formulate, Evolutionary algorithms (EAs) have attracted great research interest in recent years because EAs require no explicit formulation of the problem but only a fitness function to evaluate solutions [17]. A review of the EAs based studies is presented in Tab. 1. Despite that in all these studies empirical data was used, a large difference is shown by comparing the calibration results. The reason is that situation with a specific competitiveness level was considered for each study. For example, empirical data regarding emergency evacuation was used in [8] and [9]. However, when the parameters proposed by [9] were adopted to reproduce a real world emergency evacuation process in [8], a large bias was observed. What's more, a parameter describing the friction strength differs by a factor of about 600 in the two studies.

\section{Experiment Description and Parameter Optimisation Framework}

In this section, we first describe the pedestrian experiment where the experimental data is extracted. Then after a brief introduction of the social force model used in this study, we illustrate the DE framework for parameter optimisation.

\subsection{Pedestrian Experiment}

We conducted experiments where five participants including patient and impatient individuals proceeded through a narrow corridor. Five Japanese males participated in the 
Os
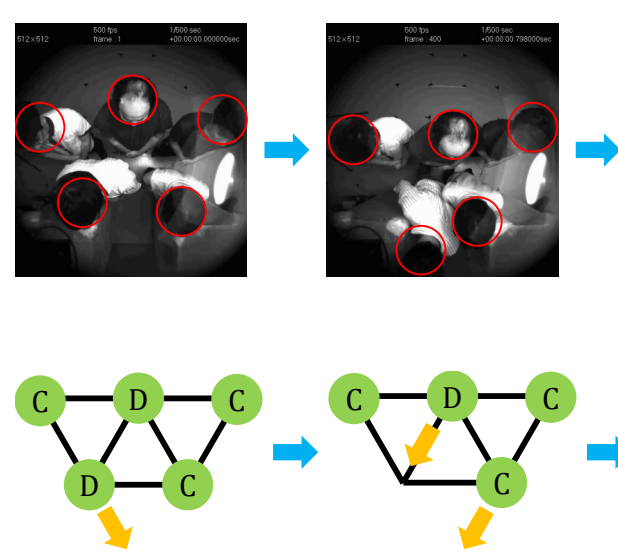

$1.6 \mathrm{~s}$

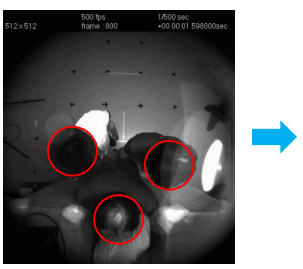

(a)
$2.4 \mathrm{~s}$

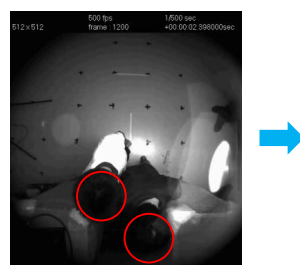

$3.2 \mathrm{~s}$

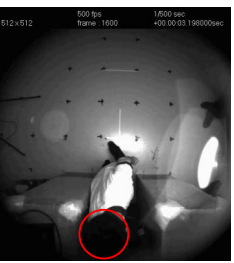

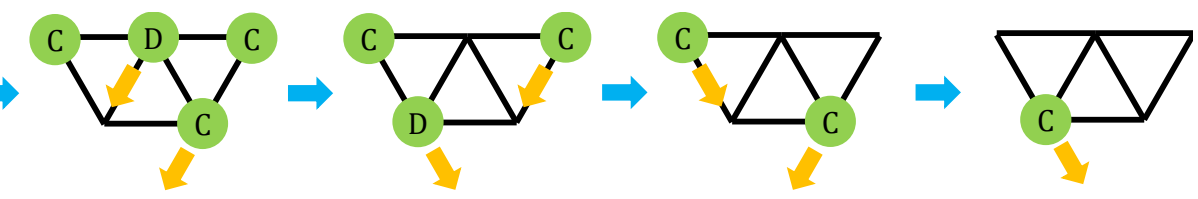

(b)

Figure 1 (a) Snapshots of the pedestrian experiment, the head of each pedestrian is marked by a red circle; (b) Predicted updating process: a co-operator (patient pedestrian) yields to other pedestrians, while a defector (impatient pedestrian) does not.

experiment. They were told to proceed through a narrow corridor before them when given a start signal. The experiment was completed when all pedestrians passed a predefined location. The competitiveness of participants was controlled by verbal instruction: the patient pedestrians are instructed to give ways to others, while the impatient pedestrians are asked to behave competitively to egress as early as possible, and soft physical body contact was allowed. The experiment was implemented several times with different configurations (initial positions of patient and impatient pedestrians). For each configuration, the experiment was conducted once. Because if the experiments were repeated for many times, the pedestrian will become familiar with the basic configuration of the experiment, thus they may behave differently than they did in the first round. A series of snapshots from the trial with the shortest evacuation time of approximately $3.3 \mathrm{~s}$ along with the predicted updating process are presented in Fig. 1, where $\mathrm{C}$ and $\mathrm{D}$ refer to the patient and impatient pedestrians, respectively. It can be seen that patient pedestrians yield to others while impatient pedestrians do not. For more details of the experiments, the authors refer to [19].

\subsection{The Social Force Model}

The social force model takes Newton's second law of dynamics as a guiding principle. In the model, each pedestrian is simulated as a Newtonian particle. Given a pedestrian $i$ of mass $m_{i}$ likes to move with a desired velocity $\mathbf{v}_{i}^{0}(t)$ in a certain direction $\mathbf{e}_{i}^{0}(t)$, the equation of motion reads:

$$
m_{i} \frac{d \mathbf{v}_{i}}{d t}=m_{i} \frac{v_{i}^{0}(t) \mathbf{e}_{i}^{0}(t)-\mathbf{v}_{i}(t)}{\tau_{i}}+\sum_{j(\neq i)} \mathbf{f}_{i j}+\sum_{W} \mathbf{f}_{i W}
$$


where $\tau_{i}$ is the relaxation time, representing the time needed for pedestrian $i$ to adapt his or her actual velocity $v_{i} . \mathbf{f}_{i j}$ represents the interaction force from other pedestrians $j$, while $\mathbf{f}_{i W}$ represents the interaction force from walls $W$. The interaction force is comprised of the social force and the body contact force. $\mathbf{f}_{i j}$ is calculated as:

$$
\mathbf{f}_{i j}=\left\{\mathbf{f}_{i j}^{\text {social }}+k g\left(r_{i j}-d_{i j}\right)\right\} \mathbf{n}_{i j}+\kappa g\left(r_{i j}-d_{i j}\right) \Delta v_{j i}^{t} \mathbf{t}_{i j}
$$

The body contact force includes body compression and friction, the strength of the two components are controlled by coefficients $k$ and $\kappa$, respectively. The compression and friction force are functional only when pedestrian $i$ touches other pedestrians $\left(d_{i j}<r_{i j}\right)$. $d_{i j}$ is the Euclidean distance between pedestrian $i$ and $j$, while $r_{i j}=r_{i}+r_{j}$ represents the radii sum of the two pedestrians. The function $g(x)$ equals zero if $x<0$, and is otherwise equal to $x . \mathbf{n}_{i j}$ denotes the normalized distance-vector pointing from pedestrian $j$ to $i, \mathbf{t}_{i j}$ denotes the tangential direction, and $\Delta v_{j i}^{t}=\left(v_{j}-v_{i}\right) \cdot \mathbf{t}_{i j}$ denotes the tangential velocity difference.

The social force describes the attempt to keep a certain safe distance to other pedestrians, it is calculated as:

$$
\mathbf{f}_{i j}^{\text {social }}=A_{i} \exp \left(\frac{-d_{i j}}{B_{i}}\right) \mathbf{n}_{i j}\left(\lambda_{i}+\left(1-\lambda_{i}\right) \frac{1+\cos \left(\mathbf{e}_{i}^{0}(t) \cdot \mathbf{n}_{i j}\right)}{2}\right)
$$

where $A_{i}$ describes the strength of interaction, $B_{i}$ corresponds to the interaction range. $d_{i j}$ represents the distance between pedestrian $i$ and $j . \lambda_{i}$ is a parameter describing the effect that the reaction of pedestrians to what happens in front of them is much stronger than to what happens behind them.

The interaction force with walls is treated analogously as: $\mathbf{f}_{i W}=\left\{A_{i} \exp \left[\left(r_{i}-d_{i W}\right) / B_{i}\right]+\right.$ $\left.k g\left(r_{i}-d_{i W}\right)\right\} \mathbf{n}_{i W}-\kappa g\left(r_{i}-d_{i W}\right)\left(\mathbf{v}_{i} \cdot \mathbf{t}_{i W}\right) \mathbf{t}_{i W}$, where $r_{i}$ represents the radii of pedestrian $i$, $d_{i W}$ is the shortest distance to wall $W, \mathbf{n}_{i W}$ denotes the direction perpendicular to wall $W$, and $\mathbf{t}_{i W}$ denotes the tangential direction.

In this study, we neglect the body contact force among pedestrians, because in the experiment strong body contact is not observed. We also neglect the social force from walls, the effect that pedestrians keep a safe distance from walls is reflected in the calculation of moving direction $\mathbf{e}^{0}$. As Fig. 2 shows, the desired velocity direction is calculated in a way that collisions with walls are evaded. For each pedestrian, circular shape representation is adopted with a radius of $0.2 \mathrm{~m}$, the mass of each pedestrian is set as $m_{i}=80 \mathrm{~kg}$.

\subsection{Parameter Optimisation}

The experimental data extracted from the experiment video was taken as reference data, which is comprised of the egress time and positions of each pedestrian. Unlike most previous experimental studies, in our experiment we did not instruct the participants to wear a hat, thus trajectory extraction using software like PeTrack does not work in our case. What is more, due to camera distortion, we consider that taking coordinate of the head as pedestrian's position would result in large deviation (please see the video in Supplementary Material for details). 


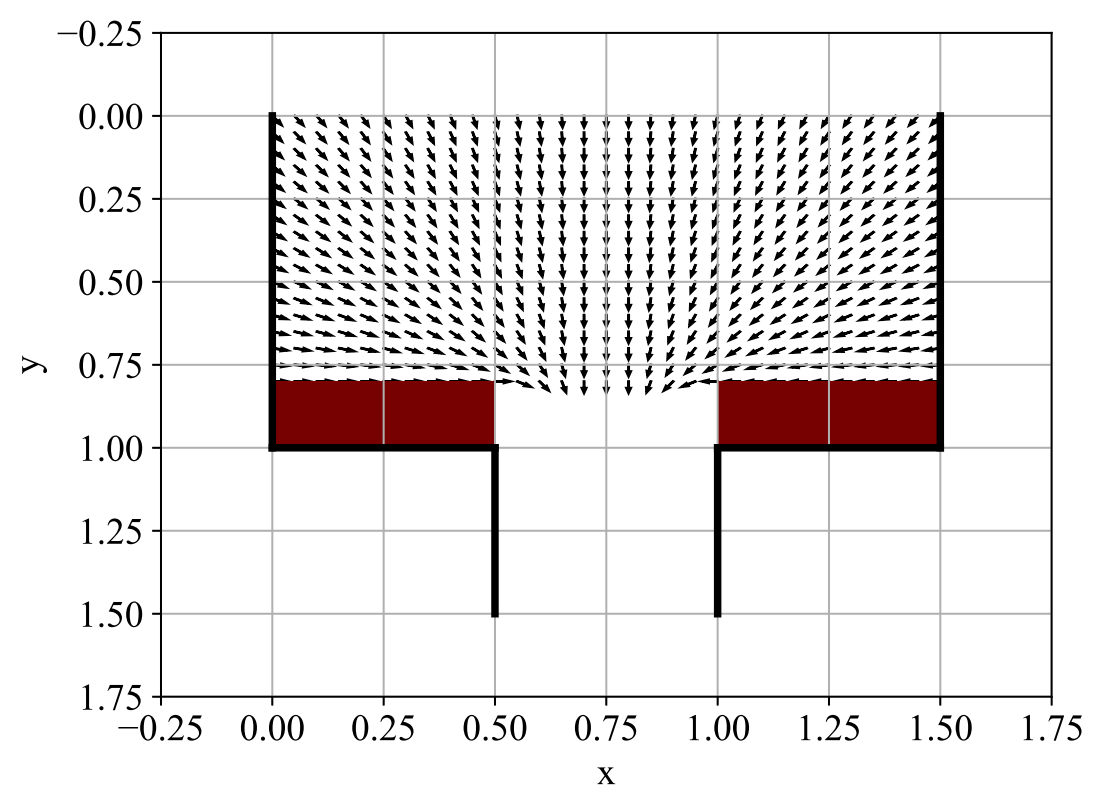

Figure 2 The potential field of the desired velocity direction. The red rectangles indicate the area where body contact with walls exists. For more detail of the method, the reader is referred to [4] and http://angel.elte.hu/pedsim/.

Hence, the data are manually extracted from the video. We made some crossing marks on the ground, the distance between two adjacent marks in the horizontal and vertical directions is $0.5 \mathrm{~m}$. We chose the upper right corner of the left obstacle as a reference point, and roughly approximated the positions of pedestrians at $1 \mathrm{~s}, 2 \mathrm{~s}$, and $3 \mathrm{~s}$ by observing the movement of their feet. We checked the video and found that the positions at these time steps could well represent the order of egress. As for the egress time of each pedestrian, we take the time point when the pedestrian body passes the line between the upper right corner of the left obstacle and the upper left corner of the right obstacle.

As the objective of parameter optimisation is to get a good match between simulation results and the reference data. We define a minimization problem to minimize the distance between simulation results and the reference data. The minimization problem is defined as follows:

$$
\begin{gathered}
\text { Minimize: } F=k_{T} \sum_{i}\left|T_{i}^{\mathrm{exp}}-T_{i}^{\operatorname{sim}}(\theta)\right|+k_{P} \sum_{t \in\{1,2,3\}} \sum_{i}\left|P_{i, t}^{\exp }-P_{i, t}^{\operatorname{sim}}(\theta)\right| \\
\text { Subject to: } \quad \theta=\left[v_{C}, v_{D}, A_{C}, A_{D}, \kappa, k, \lambda\right] \\
0<v_{C} \leq 3 \mathrm{~m} / \mathrm{s} \\
0<v_{D} \leq 3 \mathrm{~m} / \mathrm{s} \\
0<A_{C} \leq 4 \times 10^{4} \mathrm{~N} \\
0<A_{D} \leq 4 \times 10^{4} \mathrm{~N}
\end{gathered}
$$




\begin{tabular}{ll}
\hline Population size & 50 \\
Scaling factor & 0.5 \\
Crossover rate & 0.3 \\
Mutation operation & $\mathrm{DE} / \mathrm{rand} / 1$ \\
Number of iterations & 100 \\
\hline
\end{tabular}

Table 2 Parameters for DE optimisation

$$
\begin{gathered}
0<\kappa \leq 2.4 \times 10^{5} \mathrm{~kg} / \mathrm{ms} \\
0<k \leq 1.2 \times 10^{5} \mathrm{~kg} / \mathrm{m}^{2} \\
0<\lambda \leq 1
\end{gathered}
$$

In Eq. $4, T_{i}^{\exp }$ and $P_{i, t}^{\exp }$ represent the reference data. $T_{i}^{\exp }$ denotes the egress time of pedestrian $i$, while $P_{i, t}^{\exp }$ denotes the position of pedestrian $i$ at time $t . T_{i}^{\operatorname{sim}}(\theta)$ and $P_{i}^{\operatorname{sim}}(\theta)$ denote the corresponding simulation results. The experiment lasted about 3.5 $\mathrm{s}$ in real world, thus we consider the positions over three timesteps: $1 \mathrm{~s}, 2 \mathrm{~s}$, and $3 \mathrm{~s}$. Because the data were manually extracted, it is very hard to extract the whole trajectory in a detailed manner. The objective function $F$ comprises the distance in position over all pedestrians over the three timesteps and the distance in egress time over all pedestrians. $k_{T}$ and $k_{P}$ represent the weight of aforementioned two kinds of distance. Both $T_{i}^{\operatorname{sim}}(\theta)$ and $P_{i, t}^{\operatorname{sim}}(\theta)$ are functions of parameter set $\theta$, which is composed of 7 parameters. To represent the heterogeneity of competitiveness, we assign different $v$ and $A$ to patient and impatient pedestrians, e.g. $v_{c}$ represents the desired velocity of patient pedestrians while $v_{D}$ the desired velocity of impatient pedestrians. The ranges of the 7 parameters are determined according to previous study [4]. Note that two parameters $\tau$ and $B$ are not included in the parameter set $\theta$ because of the correlationship with other parameters, as shown in Eq. 1 and Eq. 3.

Since the relationship between input $\theta$ and output $F$ in the above minimization problem is hard to explicitly formulate, We apply a DE to solve this minimization problem. The parameters for DE optimisation are listed in Tab. 2, the strong global optimum search ability of such parameter combination is verified in [20].

\section{Results and Analysis}

The role of the reference data is to describe the desired behavior that the simulation should generate. To reflect different levels of emphasis on the two kinds of distance in Eq. 4. The DE based optimisation was conducted for five different combinations of weights: $\left(k_{T}, k_{P}\right) \in\{(0,1),(1,0),(1,0.05),(1,0.1),(1,0.5)\}$. e.g. $\left(k_{T}, k_{P}\right)=(0,1)$ means only considering the distance of positions and neglect the distance of egress time. We first completed the first two implementations to investigate the scale of distance in terms of egress time and positions separately. Then based on the convergence value of the objective 


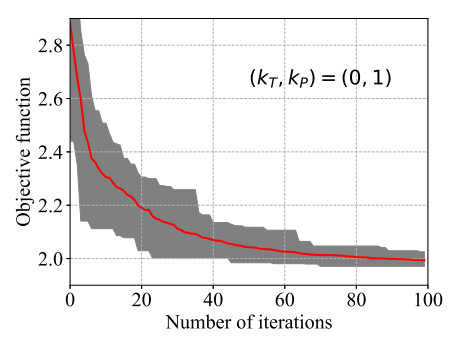

(a)

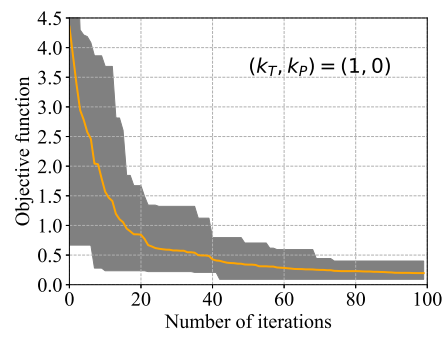

(b)

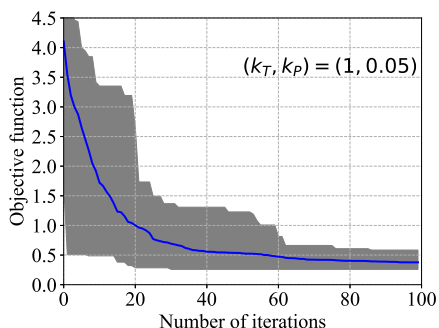

(c)

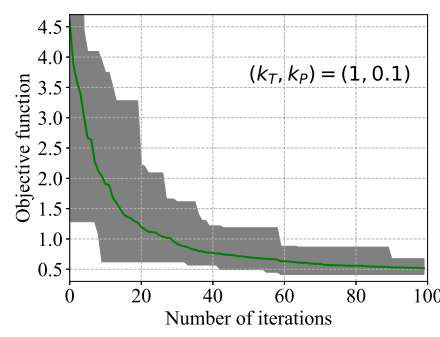

(d)

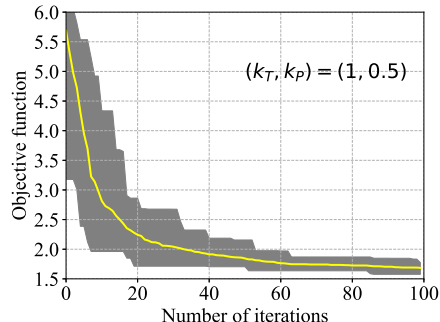

(e)

Figure 3 The convergence curves of optimisation using DE for five different combinations of $k_{T}$ and $k_{P}$ : the solid line indicates the average of 30 times implementations, the gray shadow shows upper and lower bound.

function (as shown in Fig. 3. (a) and (b)), set the weight value in the last three cases. It is observed that the scale of the distance of positions is much larger than egress time. For each combination, the DE implementation was run 30 times. The convergence curves are shown in Fig. 3, in the figure it is observed that in all the five situations the algorithm almost converges before the maximum number of iterations, indicating that 100 times iterations are enough for reaching a near-optimal solution.

The optimal solutions based on combination $\left(k_{T}, k_{P}\right)=(1,0.1)$ are shown in Tab. 3 . We only present the results of the desired velocity and the strength of interaction because the heterogeneity of competitiveness, which is our main focus in this study, is represented by these two parameters. In the table, it is observed that rather than just one unique combination of parameters, many different combinations in the parameter space can produce similar results in terms of good fitness (small value of $F$ in Eq. 4). For example, the value of objective function $F$ is the same $(F=0.50)$ in the $16^{\text {th }}, 27^{\text {th }}$, and $28^{\text {th }}$ implementations. But the values of calibrated parameters vary a lot.

In previous studies [14][15], pedestrian competitiveness is simulated by adjusting the value of $v^{0}$ and $A$, it is assumed that the desired velocity of patient pedestrians is lower than impatient pedestrians, and the strength of interaction of patient pedestrians is stronger. However, in Tab. 3 all the results do not support this assumption. To further investigate the statistical characteristics of the solutions found by DE, we take the average of 30 times implementations and present the results in Fig. 4. In the figure, it is observed that the average values, which are indicated by green triangles, are in good consistent with the assumption in previous studies. What is more, the deviations in the last four cases 


\begin{tabular}{|c|c|c|c|c|c|c|c|c|c|c|}
\hline Parameters & $1 \mathrm{st}$ & 2 nd & $3 \mathrm{rd}$ & 4 th & 5 th & 6th & 7 th & 8 th & 9th & 10th \\
\hline$v_{D}(\mathrm{~m} / \mathrm{s})$ & 1.84 & 1.85 & 1.88 & 2.26 & 2.34 & 1.79 & 2.65 & 1.96 & 2.19 & 2.04 \\
\hline$A_{D}(N)$ & 3836.42 & 4953.81 & 3619.61 & 7721.18 & 6594.43 & 4773.69 & 7048.43 & 5080.40 & 4242.31 & 4668.11 \\
\hline$F$ & 0.64 & 0.66 & 0.59 & 0.67 & 0.35 & 0.64 & 0.52 & 0.57 & 0.57 & 0.80 \\
\hline$v_{C}(m / s)$ & 1.56 & 1.85 & 1.58 & 2.62 & 2.15 & 1.93 & 2.09 & 1.63 & 1.63 & 2.10 \\
\hline$v_{D}(\mathrm{~m} / \mathrm{s})$ & 2.04 & 2.30 & 2.23 & 2.41 & 1.74 & 2.29 & 2.44 & 1.87 & 2.67 & 2.04 \\
\hline$A_{C}(N)$ & 4688.11 & 7921.72 & 3951.08 & 11482.46 & 8152.11 & 12636.50 & 7153.41 & 5461.41 & 2591.87 & 10960.06 \\
\hline$A_{D}(N)$ & 5520.53 & 4923.98 & $\mathbf{5 7 9 3 . 8 5}$ & 6698.43 & 2575.16 & 8339.67 & 6818.45 & 3355.76 & 6847.58 & 5015.48 \\
\hline$F$ & 0.53 & 0.57 & 0.44 & 0.52 & 0.59 & 0.50 & 0.44 & 0.51 & 0.41 & 0.45 \\
\hline$A_{C}(N)$ & 4539.13 & 2330.41 & 8035.00 & 8558.71 & 819.33 & 12343.75 & 1752.26 & 7525.53 & 11666.11 & 3122.77 \\
\hline$A_{D}(N)$ & 4721.20 & 8855.34 & 4544.99 & 9039.20 & 2797.74 & 5424.42 & 5318.98 & 4092.57 & 5419.69 & 4191.19 \\
\hline$F$ & 0.47 & 0.59 & 0.51 & 0.67 & 0.56 & 0.42 & 0.50 & 0.50 & 0.54 & 0.48 \\
\hline
\end{tabular}

Table 3 Results of 30 times DE implementations based on combination $\left(k_{T}, k_{P}\right)=(1,0.1)$, the number with larger value is bolded for comparison.

are very large, indicating that many different combinations of parameters could produce similar outcomes in terms of good fitness.

To show the robustness of our method, we also present the results based on $m_{i}=60 \mathrm{~kg}$ as a reference. In Fig. 4, it is observed that the general conclusion $\left(v_{C}^{0}<v_{D}^{0}, A_{C}>A_{D}\right)$ is the same as Fig. 4. The results are also very similar quantitatively for $v^{0}$. As for $A$, apparently the values in the $60 \mathrm{~kg}$ case are smaller. This difference is not difficult to understand, divide both sides of Eq. 1 by $m_{i}$, one can see that $A$ is linearly correlated to $m_{i}$, while $v^{0}$ and $m_{i}$ are independent of each other (please see Eq. 3 and Eq. 2 for details).

Furthermore, in Fig. 4 it is observed that depending on the combination of weights, the results varied greatly. Especially, in the case where only the positions are considered as reference data $\left(k_{T}=0, k_{P}=1\right)$, the results are very different from others: not only the average is different from others, but also the deviation is relatively small. To investigate the optimal weight combination, additional reference data as a test set is needed, this is beyond the scope of this study. Nonetheless, one can observe that the selection of reference data has a strong impact on the calibration results.

A sensitivity analysis is conducted to identify the important parameters of the model. The main idea is to measure the importance of a parameter by its randomness. If a parameter has small randomness, it is likely to be an important parameter [18]. We use the coefficient of variation to measure the randomness of a parameter. Given a parameter, its coefficient of variation is calculated as $C V=\frac{\sigma}{\mu}, \sigma$ is the standard deviation, while $\mu$ refers to the average value. The results for the five combinations of weights are presented in Fig. 6. It is observed that generally, the desired velocity plays a dominant role, while the effect of $k$ and $\lambda$ is relatively weak. What is more, if the distance of egress time is included in the objective function, the results, as shown in the last four cases, are 


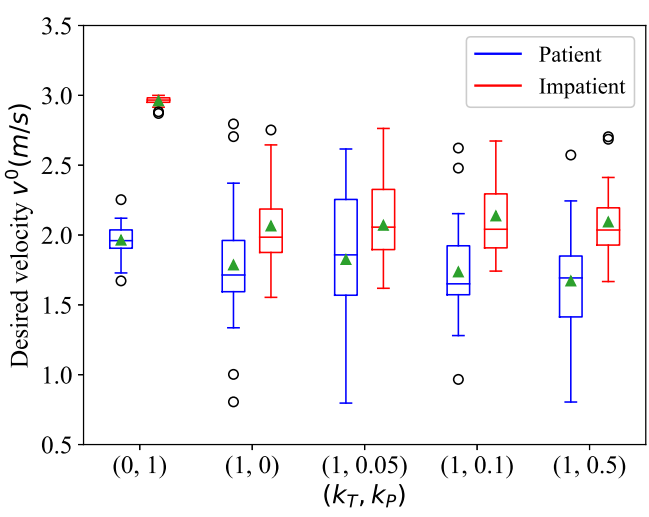

(a)

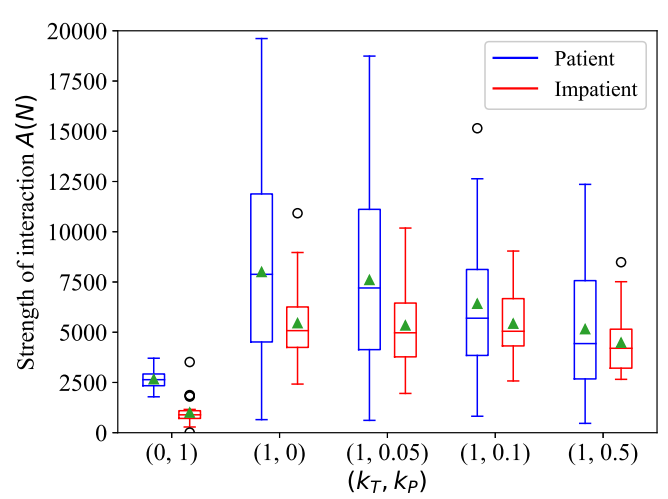

(b)

Figure 4 Comparison of DE optimisation results for the patient and impatient pedestrians. The average values are marked by green triangles. (a) Comparison of $v^{0}$; (b) Comparison of $A$.

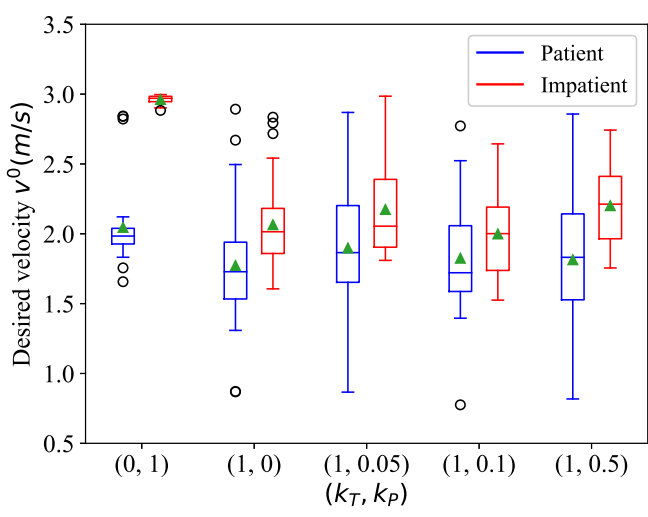

(a)

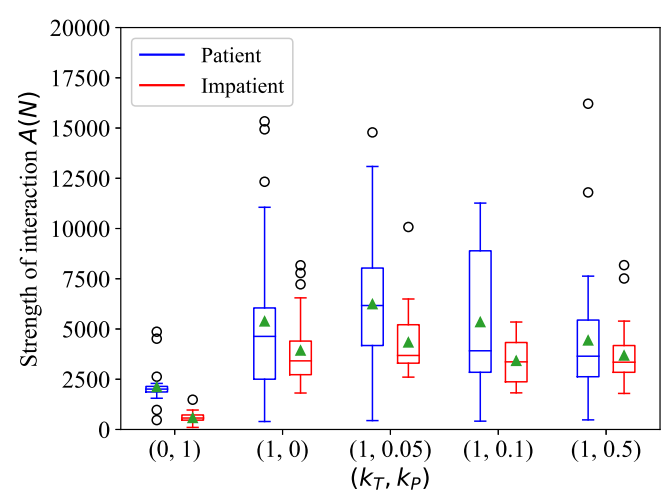

(b)

Figure 5 DE optimisation results for the reference group $\left(m_{i}=60 \mathrm{~kg}\right)$. The simulation setup is the same as Fig. 4 except for that the mass of pedestrian is different. 


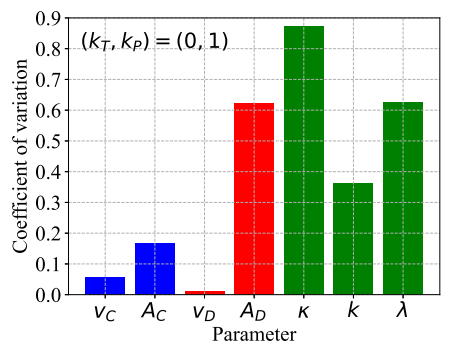

(a)

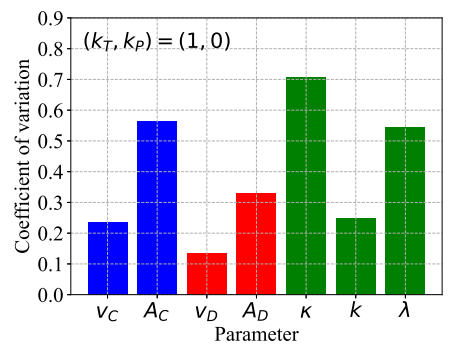

(b)

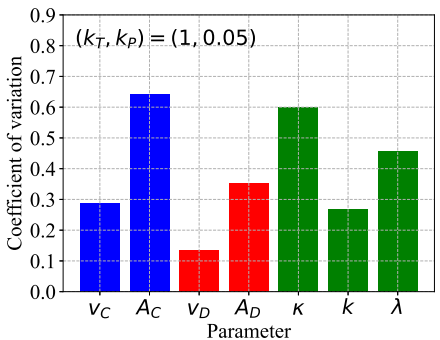

(c)

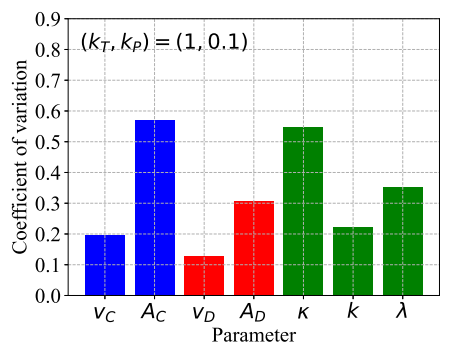

(d)

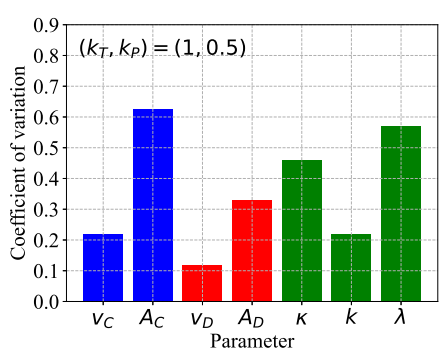

(e)

Figure 6 The coefficient of variation of the seven parameters of 30 times implementations.

significantly different from the first one. We consider a trade-off relationship between reproducing the behaviour of egress time and positions. Adding some complex updating rules such as the ellipse body shape representation, body rotation of pedestrian, and pedestrian vision might improve the overall performance.

\section{Conclusion}

In this study, we aim to address the problem of pedestrian competitiveness representation using the social force model. Starting from an assumption that higher competitiveness could be represented by higher desired velocity and weaker interaction strength, we conducted a small scale pedestrian experiment where a group is comprised of patient and impatient pedestrians. A DE is incorporated in the social force model to determine the optimal combination of parameters. The results are in good consistency with the aforementioned assumption.

In the literature of experimental verification of the 'faster-is-slower' effect, a mixture of evidence is observed. The cause of this problem might be that pedestrian competitiveness was hard to quantitatively control. In most related studies participants were verbally instructed to behave 'competitively' or 'non-competitively', we consider that the level of pedestrian competitiveness differs according to different research groups. What is more, since under laboratory conditions participants are conscious that they are not facing a real evacuation. The potential of experimental studies to reflect reality is limited. Our DE-based approach could be considered as a quantitative way to represent pedestrian 
competitiveness. The level of competitiveness is quantitatively represented by the parameters in the social force model in our method. Therefore, one can compare the level of competitiveness in different situations with the same criteria.

This study stresses the importance of model calibration. In the research field of pedestrian simulation, compared to model calibration, much more effort are contributed to model improvement and extension. However, in this paper, it is shown that through appropriately adjusting the parameters, considerable accuracy could be achieved using only a simple version of the social force model.

We found that the selection of reference data has a strong impact on the calibration results. In a previous study, it is stated that the importance of each parameter differs for different scenarios [18]. In this study, we found that even for the same scenario, this effect exists depending on the objective behaviour to reproduce. What is more, a trade-off relationship exists in the reproduction of two kinds of behaviours in terms of pedestrian positions and egress time. Here we propose a new question: to what extent can the social force model reproduce real human behaviour? To answer this question, not only the parameters but also the updating rules could be encoded into the solution vector. Using the open-access data archive as reference data, in a specific situation, the importance of each parameter and updating rule could be investigated, along with parameter calibration. We will leave this for future work.

Acknowledgements This work is supported by China Scholarship Council, JST-Mirai Program Grant Number JPMJMI20D1, Japan, JSPS KAKENHI Grant Number JP21H01570 and 21H01352, Japan, and partially supported by FONDAZIONE CARIPLO (Italy), "LONGEVICITY - Social Inclusion for the Elderly through Walkability", Rif. 2017-0938.

\section{References}

[1] Haghani, M., Sarvi, M., Shahhoseini, Z.: When 'push' does not come to 'shove': Revisiting 'faster is slower' in collective egress of human crowds. Transportation Research Part A: Policy and Practice 122, 51-69 (2019). doi:10.1016/j.tra.2019.02.007

[2] Chen, X., Treiber, M., Kanagaraj, V., Li, H.: Social force models for pedestrian traffic - state of the art. Transport Reviews 38(5), 625-653 (2017). doi:10.1080/01441647.2017.1396265

[3] Helbing, D., Molnár, P.: Social force model for pedestrian dynamics. Physical Review E 51(5), 4282-4286 (1995). doi:10.1103/physreve.51.4282

[4] Helbing, D., Farkas, I., Vicsek, T.: Simulating dynamical features of escape panic. Nature 407(6803), 487-490 (2000). doi : 10 . $1038 / 35035023$

[5] Haghani, M., Sarvi, M.: Simulating pedestrian flow through narrow exits. Physics Letters A 383(2-3), 110-120 (2019). doi:10.1016/j.physleta.2018.10.029 
[6] Sticco, I., Frank, G., Cerrotta, S., Dorso, C.: Room evacuation through two contiguous exits. Physica A: Statistical Mechanics and its Applications 474, 172-185 (2017). doi:10.1016/j.physa.2017.01.079

[7] JOHANSSON, A., HELBING, D., SHUKLA, P.K.: SPECIFICATION OF THE SOCIAL FORCE PEDESTRIAN MODEL BY EVOLUTIONARY ADJUSTMENT TO VIDEO TRACKING DATA. Advances in Complex Systems 10(supp02), 271288 (2007). doi:10.1142/s0219525907001355

[8] Sticco, I., Frank, G., Dorso, C.: Social force model parameter testing and optimization using a high stress real-life situation. Physica A: Statistical Mechanics and its Applications 561, 125299 (2021). doi:10.1016/j.physa.2020.125299

[9] Li, M., Zhao, Y., He, L., Chen, W., Xu, X.: The parameter calibration and optimization of social force model for the real-life 2013 ya' an earthquake evacuation in china. Safety Science 79,243-253 (2015). doi:10.1016/j.ssci.2015.06.018

[10] Garcimartín, A., Zuriguel, I., Pastor, J., Martín-Gómez, C., Parisi, D.: Experimental evidence of the "faster is slower" effect. Transportation Research Procedia 2, 760767 (2014). doi:10.1016/j.trpro.2014.09.085

[11] Pastor, J.M., Garcimartín, A., Gago, P.A., Peralta, J.P., Martín-Gómez, C., Ferrer, L.M., Maza, D., Parisi, D.R., Pugnaloni, L.A., Zuriguel, I.: Experimental proof of faster-is-slower in systems of frictional particles flowing through constrictions. Physical Review E 92(6), 062817 (2015). doi:10.1103/physreve.92.062817

[12] Daamen, W., Hoogendoorn, S.P.: Emergency door capacity: Influence of door width, population composition and stress level. Fire Technology 48(1), 55-71 (2010). doi:10.1007/s10694-010-0202-9

[13] Cao, R.F., Lee, E.W.M., Yuen, A.C.Y., Chen, T.B.Y., Cordeiro, I.M.D.C., Shi, M., Wei, X., Yeoh, G.H.: Simulation of competitive and cooperative egress movements on the crowd emergency evacuation. Simulation Modelling Practice and Theory 109, 102309 (2021). doi:10.1016/j.simpat.2021.102309

[14] von Schantz, A., Ehtamo, H.: Pushing and overtaking others in a spatial game of exit congestion. Physica A: Statistical Mechanics and its Applications 527, 121151 (2019). doi:10.1016/j.physa.2019.121151

[15] Heliövaara, S., Ehtamo, H., Helbing, D., Korhonen, T.: Patient and impatient pedestrians in a spatial game for egress congestion. Physical Review E 87(1), 012802 (2013). doi:10.1103/physreve.87.012802

[16] Wolinski, D., Guy, S.J., Olivier, A.H., Lin, M., Manocha, D., Pettré, J.: Parameter estimation and comparative evaluation of crowd simulations. Computer Graphics Forum 33(2), 303-312 (2014). doi : 10 .1111/cgf. 12328 
[17] Zhong, J., Hu, N., Cai, W., Lees, M., Luo, L.: Density-based evolutionary framework for crowd model calibration. Journal of Computational Science 6, 11-22 (2015). doi:10.1016/j.jocs.2014.09.002

[18] Zhong, J., Cai, W.: Differential evolution with sensitivity analysis and the powell's method for crowd model calibration. Journal of Computational Science 9, 26-32 (2015). doi:10.1016/j.jocs.2015.04.013

[19] Cui, G., Yanagisawa, D., Nishinari, K.: Incorporating genetic algorithm to optimise initial condition of pedestrian evacuation based on agent aggressiveness. Physica A: Statistical Mechanics and its Applications 583, 126277 (2021). doi:10.1016/j.physa.2021.126277

[20] Qin, A., Huang, V., Suganthan, P.: Differential evolution algorithm with strategy adaptation for global numerical optimization. IEEE Transactions on Evolutionary Computation 13(2), 398-417 (2009). doi:10.1109/tevc.2008.927706 